This item was submitted to Loughborough's Research Repository by the author.

Items in Figshare are protected by copyright, with all rights reserved, unless otherwise indicated.

\title{
Equivalent circuit analysis for 3D metamaterials with fringing field correction
}

\section{factor}

PLEASE CITE THE PUBLISHED VERSION

https://doi.org/10.1049/cp.2017.0248

PUBLISHER

IET

VERSION

AM (Accepted Manuscript)

\section{PUBLISHER STATEMENT}

This work is made available according to the conditions of the Creative Commons Attribution-NonCommercialNoDerivatives 4.0 International (CC BY-NC-ND 4.0) licence. Full details of this licence are available at: https://creativecommons.org/licenses/by-nc-nd/4.0/

\section{LICENCE}

CC BY-NC-ND 4.0

\section{REPOSITORY RECORD}

Whittaker, T., W.G. Whittow, and J.C. Vardaxoglou. 2019. "Equivalent Circuit Analysis for 3D Metamaterials with Fringing Field Correction Factor". figshare. https://hdl.handle.net/2134/37290. 


\title{
Equivalent Circuit Analysis for 3D Metamaterials with Fringing Field Correction Factor
}

\author{
T. Whittaker*, W. Whittow ${ }^{\dagger}$ and Y. Vardaxoglou ${ }^{\#}$ \\ Wolfson School of Mechanical, Electrical and Manufacturing Engineering \\ Loughborough University \\ Loughborough, UK \\ *t.whittaker@lboro.ac.uk, ${ }^{+}$w.g.whittow@lboro.ac.uk, ${ }^{\#}$ j.c.vardaxoglou@lboro.ac.uk
}

Keywords: Equivalent Circuit, Metasurface, Metamaterials, Square Loop, Fringing Fields.

\begin{abstract}
This paper presents a fringing field correction factor for circuit modelling techniques of metasurfaces and metamaterials. The theory is then applied to periodic three dimensional metamaterial meta-atoms. Equivalent circuits have been validated using CST Microwave Studio.
\end{abstract}

\section{Introduction}

Equivalent circuit modelling theory can be a powerful tool in predicting performance of electromagnetic components. It has significant key advantages over full wave simulations, such as being less computationally intensive and the ability to simulate equivalent circuits alongside conventional electronic circuitry. The equivalent circuit method uses solutions for infinite periodic metallic obstacles in a plane wave [1]. This method has been used before by creating 2D metasurface equivalent circuits for square rings [2], Jerusalem crosses [3] and gridded squares [4]. These examples [2-4] consist of infinitely thin obstacles. Solutions for obstacles with finite thickness will be used in this paper to model 3D meta-atom structures. Theory for fringing field compensation will be developed in Section 2 to improve accuracy of the circuit model.

\section{Correction Factor}

For conventional microstrip transmission lines Wheeler [5] compensates for fringing fields with a width adjustment $\Delta w$. Equation (1) can be applied to metamaterials/metasurfaces if the law of images is followed. Where $w$ is the microstrip width, $t$ is the microstrip height/thickness and $h$ is the height between microstrip and ground plane [5]:

$$
R(t, w, h)=\Delta w=\frac{t}{\pi} \ln \frac{4 e^{1}}{\sqrt{\left(\frac{t}{h}\right)^{2}+\left(\frac{1 / \pi}{w / t+1.10}\right)^{2}}}
$$

\section{3D Metamaterial Equivalent Circuits}

The meta-atom modelled is shown in Figure 1. It consists of two square loops of width $L$ with square cross-sectional wire of thickness $W$ and a bridge connection along the top edge of length $L$. The inside length of the loop is $T$ and the metamaterial has a unit cell periodicity of $P$ in the $\mathrm{x}$ and $\mathrm{y}$ axis. The conductor is assumed to be lossless (PEC) in a host of free space. The unit cell is illuminated with a vertically polarized (y-axis) TEM plane wave propagating along the $\mathrm{z}$ axis.

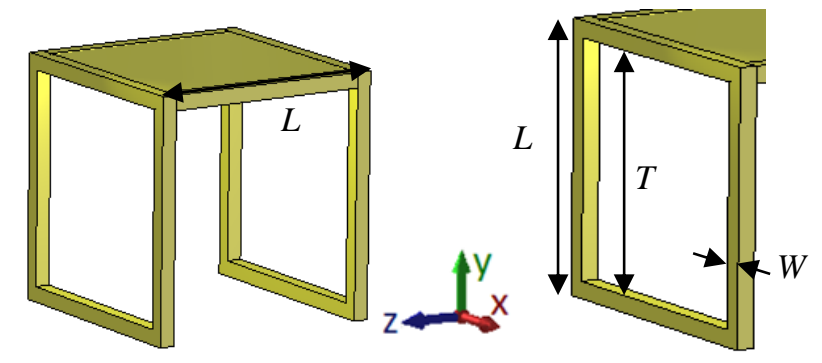

Figure 1. Unit cell of square loop pair with bridge. Metaatoms are assumed to be infinite in extent in $\mathrm{x}$ and $\mathrm{y}$ axes

The solutions to calculate equivalent circuit values for rectangular cross-section posts can be found in [1, pp. 285$286]$ and have been omitted here due to space constraints. Equations in [1] are referred to as $X_{n}\left(a, d^{\prime}, d^{\prime \prime}, \lambda\right)$ for equivalent reactance circuit components for vertical inductive posts and $B_{n}\left(a, d^{\prime}, d^{\prime \prime}, \lambda\right)$ for equivalent susceptance circuit components for horizontal capacitive posts. The arguments $a, d^{\prime}, d^{\prime \prime}$ and $\lambda$ corresponded to the post periodicity, the post width, the post depth and the wavelength of the source plane wave respectively.

Equations (2-5) are modified from [1] and reflect the conversion of infinite length posts to finite objects. Equations (2-4) are the equivalent circuit components for the capacitance between vertical adjacent loops; inductance of the vertical loop arms; and self-capacitance inside the loop. Equation (5) corresponds to the equivalent circuit components for the bridge connection between the loop pairs. Equations (2) and (5) include the fringing field correction factor.

$$
\begin{gathered}
B_{n \_l o o p}=B_{n}(P, L, W, \lambda) \frac{L+\Delta W}{P} \\
X_{n \_l o o p}=X_{n}(P, 2 W, W, \lambda) \frac{L}{P} \\
B_{n \_ \text {gap }}=B_{n}(L-W, W, W, \lambda) \frac{L-2 W}{P} \\
B_{n_{-} \text {con }}=j B_{n}(P, W, T, \lambda) \frac{L+\Delta W}{P}
\end{gathered}
$$

Capacitive coupling between the two square loops is not captured in the above equations and can be approximately modelled using the classic capacitor equation, $C=\varepsilon_{0} A / d$. 


$$
C=\frac{\varepsilon_{0} A}{T}
$$

Where $\varepsilon_{0}$ is the permittivity of free space and $A$ is the surface area of the square loops that face each other. The capacitance value can then be converted into a susceptance value where $\omega$ is angular frequency of the incoming wave:

$$
B_{\text {couple }}=C \omega
$$

Equations from [1] are in the form of T-networks of reactive components and can be constructed together in layers. The first and third layers are square loops; the components relating to the square loop are lumped into a single effective T-network. The two layers are then connected together with the coupling element in equation (7). The second layer consists of the bridge connection with the included width correction. This gives the resulting equivalent circuit shown in Figure 2. $Z_{\text {loop }}$ is defined in equation (8) as the summation of loop reactances for the reason of space constraints.

$$
Z_{\text {loop }}=\frac{-j}{B_{\text {a_gap }}}+\frac{-j}{B_{a_{\_} \text {loop }}}+j X_{b_{-} \text {loop }}
$$

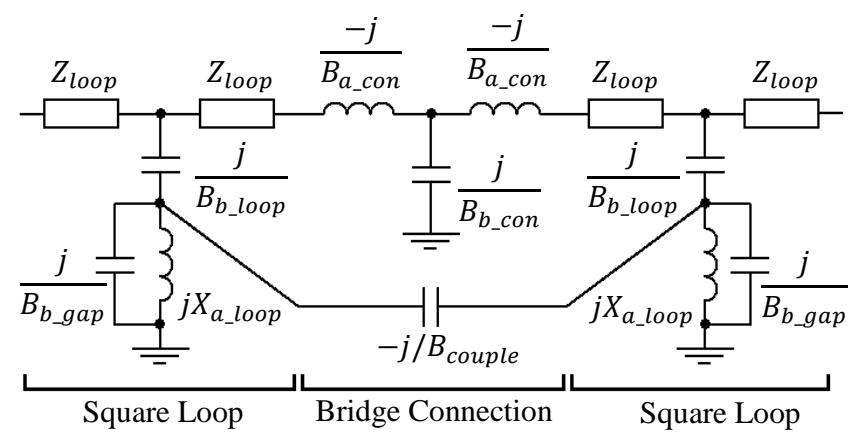

Figure 2. Equivalent circuit of double square loop with bridge

Figure 3 shows the simulation and equivalent circuit scattering parameters for the metamaterial. The response of the equivalent circuit is calculated in MATLAB by using conventional circuit theory. The circuit is condensed into a single $\mathrm{T} / \pi$ impedance network before being converted into a transmission (ABCD) matrix, from here S-parameters can be easily extracted [6]. The circuit is fed with ports of reference impedance of free space $(377 \Omega)$. As can be seen, both sets of graphs display good agreement between simulations and equivalent circuits. However the circuit is most accurate at the lower frequencies. The connected square loops possess a wide band-stop response; by increasing the conductor thickness the bandwidth can be further increased.

\section{Conclusion}

This paper has shown that current 2D metasurface equivalent circuit modelling techniques can also be applied to 3D metaatom metamaterials with good agreement to simulation results. This modelling technique is particularly advantageous as the geometry is described within the equations which allow for easy re-computation for dimensions adjustments. Future work will use this model to allow for fast optimisation and exploration of 3D meta-atoms.
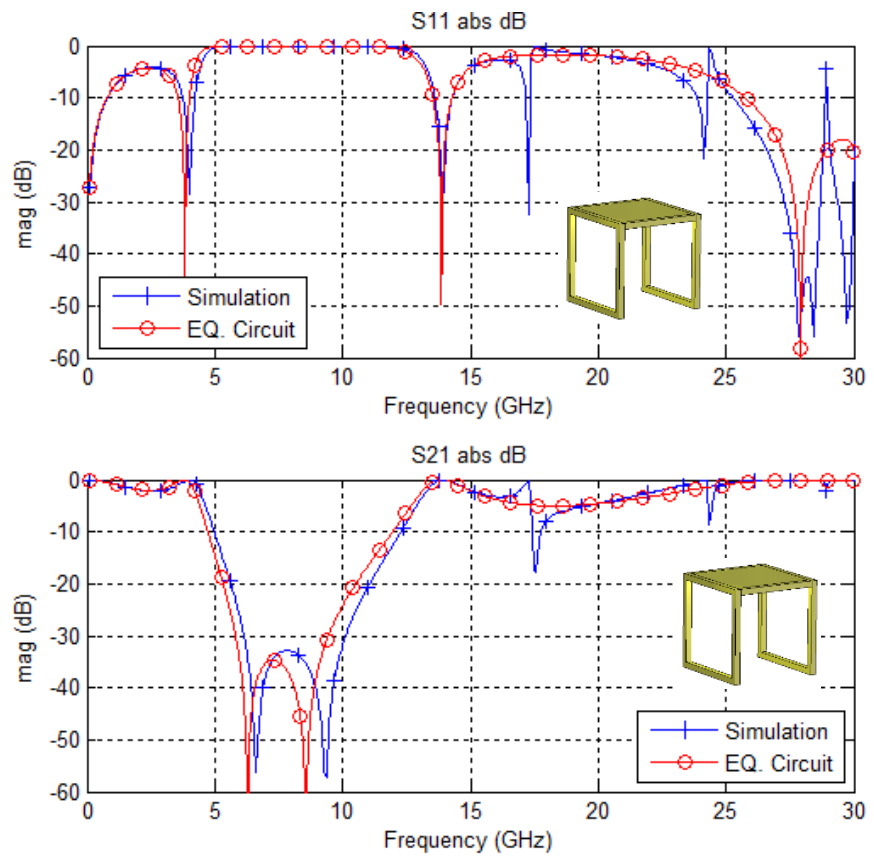

Figure 3. S-parameter comparison of simulated and calculated results of bridged double square loops. ( $T=0.5 \mathrm{~mm}, L=9 \mathrm{~mm}$, $P=10 \mathrm{~mm})$

\section{Acknowledgements}

This work was supported by the UK Engineering and Physical Sciences Research Council (EPSRC) and is part of the SYMETA project (https://www.symeta.co.uk/). Grant reference code EP/N010493/1.

\section{References}

[1] N. Marcuvitz, Waveguide Handbook. London: Peter Peregrinus Ltd, 1986.

[2] R. J. Langley and E. A. Parker, "Equivalent circuit model for arrays of square loops," Electron. Lett., vol. 18, no. 7, p. 294, 1982.

[3] I. Anderson, "On the Theory of Self-Resonant Grids," Bell Syst. Tech. J., vol. 54, no. 10, pp. 1725-1731, Dec. 1975.

[4] C. K. Lee and R. J. Langley, "Equivalent-circuit models for frequency-selective surfaces at oblique angles of incidence," IEE Proc. H Microwaves, Antennas Propag., vol. 132, no. 6, p. 395, 1985.

[5] H. A. Wheeler, "Transmission-Line Properties of a Strip on a Dielectric Sheet on a Plane," IEEE Trans. Microw. Theory Tech., vol. 25, no. 8, pp. 631-647, Aug. 1977.

[6] D. A. Frickey, "Conversions between S, Z, Y, H, $\mathrm{ABCD}$, and $\mathrm{T}$ parameters which are valid for complex source and load impedances," IEEE Trans. Microw. Theory Tech., vol. 42, no. 2, pp. 205-211, 1994. 